\title{
ARTIGOS
}

\section{INFLUÊNCIA DE CORTICÓIDE, NA DOENÇA DE CHAGAS CRÔNICA, ADMINISTRADO EM VIRTUDE DE AFECÇÕES ASSOCIADAS}

\author{
Anis Rassi, Vicente Amato Neto, Astolpho Ferraz de Siqueira, \\ Jarbas Doles, Maurício Sérgio Brasil Leite, Osterno Queiroz Silva \\ e Vasco Martins Cardoso
}

\begin{abstract}
Individuos acometidos de doença de Chagas em fase crônica foram tratados com corticóide, em virtude de afecções concomitantes. A fim de avaliar repercussões sobre a infecção devida ao Trypanosoma cruzi, houve adoção de procedimentos de várias ordens, representados por métodos referentes à parasitose, exames inespecíficos e subsídios clínico, eletrocardiográfico e radiológico, tendo também sido mantido, para comparação, grupo controle. Através do xenodiagnóstico ficou constatada acentuação da parasitemia, diretamente relacionada com a dose de corticóide, sem influência na evolução da enfermidade parasitária, pelo menos durante o período no qual ocorreu a investigação.
\end{abstract}

Palavras-chaves: Doença de Chagas crônica. Corticóide.

O comportamento da infecção humana pelo Trypanosoma cruzi quando vigente imunodeficiência ainda requer melhores esclarecimentos. Essa necessidade está ficando cada vez mais imperiosa porque a concomitância de doença de Chagas e afecções nas quais o distúrbio imunitário existe é atualmente mais comum e, também, em virtude do maior uso atual de fármacos que originam a imunodepressão ${ }^{2}{ }^{10}$. Além disso, a crescente realização de transplantes de órgãos, sendo preciso coibir rejeições, envolve hoje, em várias oportunidades, pacientes acometidos pela enfermidade referida, figurando eles como doadores ou receptores ${ }^{6811}$.

O número de relatos de investigações experimentais, sobre a atuação de corticóide, desenvolvidas nos modelos baseados em diferentes tipos de animais, representados sobretudo por camundongos, é razoável. A despeito de não haver unanimidade, as deduções indicam comumente aumentos da parasitemia, do parasitismo tecidual e da mortalidade, revelando também mudança do tropismo em relação aos órgãos que o T. cruzi infecta e atenuação do contingente inflamatório.

Departamento de Clínica Médica da Faculdade de Medicina da Universidade Federal de Goiás, Goiânia, Go.

Endereço para correspondência: Prof.Anis Rassi. Av. A n ${ }^{\circ} 333$ (Setor Oeste), 74110-020 Goiânia, GO, Brasil.

Recebido para publicação em 22/01/96.
Tais estudos focalizaram as fases aguda, pósaguda imediata e crônica, tendo apontado, além do que já citamos, comportamentos diversos de cepas ${ }^{13512} 13$. Nessas pesquisas, diferentes modalidades de corticóide foram utilizadas, isoladamente ou em associação com compostos tripanossomicidas.

No que concerne a pacientes com doença de Chagas, reativações vêm sendo comprovadas quando recebem coração por meio de transplante, corticóide fazendo parte da tática imunossupressora, ou em virtude da administração de certos fármacos para tratamento de leucemias, acrescentando-se a isso, hoje em dia, a participação da síndrome da imunodeficiência adquirida (AIDS) 4 $^{4} 9^{1011}$. Quanto ao transplante de rim, duas situações estão demarcadas: desenvolvimento de fase aguda da doença, clínica e laboratorialmente manifesta, se o órgão procede de doador infectado, e ausência deste acontecimento caso o receptor já tenha a parasitose ${ }^{68}$.

Diante do exposto, torna-se extremamente conveniente, através de investigações experimentais e de casuísticas bem analisadas, abordar intensamente o assunto, sobretudo a fim de possibilitar tarefas assistenciais apropriadas. Por isso, tentamos contribuir por meio de avaliação do que sobreveio em indivíduos infectados pelo T. cruzi, quando corticóide foi empregado judiciosamente em contexto terapêutico, comparecendo a moléstia 
Rassi A, Amato Neto V, Siqueira AF, Doles J, Leite MSB, Silva OQ, Cardoso VM. Influência de corticóide, na doença de Chagas crônica, administrado em virtude de afecções associadas. Revista da Sociedade Brasileira de Medicina Tropical 30:93-99, mar-abr, 1997.

parasitária como acontecimento associado. Praticamos apreciações, específicas ou não, paralelamente, e estipulamos indispensável controle.

\section{MATERIAL E MÉTODOS}

Dispusemos os componentes da casuística em três Grupos (I, II e III). Provas sorológicas prévias identificaram a presença da doença de Chagas em todos, correspondendo ela às formas clínicas indeterminada, não-cardíaca ou cardíaca sem descompensação.

Houve prescrição de quatro tipos de corticóide (triancinolona, betametasona, prednisona e dexametasona), cuja escolha dependia do médico responsável pelo caso. Quanto ao Grupo I, as doses afiguraram-se variáveis, derivando de ações médicas costumeiras, mas, a propósito do III, recorremos a iniciativas conforme as quais ocorreu, na medida do possível, uma certa uniformização. Os indivíduos do Grupo II, estabelecidos como controle, não receberam nenhum medicamento.

A Tabela 1 resume as características básicas dos pacientes nos 3 grupos estudados.

\section{Grupo I}

Casuística. Composta de 10 pacientes, sendo 6 do sexo masculino e 4 do feminino. As idades variaram de 16 a 65 anos (média de $37,6 \pm 16,1)$. As afecções associadas que justificaram o uso de corticóide foram: pênfigo foliáceo (5), colestase intra-hepática (1), córiocarcinoma do endométrio (1), doença reumática (1), taquiarritmia ventricular em estado de mal (1) e doença de Hodgkin (1).

Uso de corticóide. As doses dos diferentes corticóides, computadas como de prednisona e calculadas como médias, variaram de 125 a $5 \mathrm{mg}$, com administração progressivamente decrescente, de 12 em 12 horas, e em etapas que duraram de 45 a 120 dias.

Exames auxiliares. Xenodiagnóstico com 20 ninfas de terceiro ou quarto estádios do Triatoma infestans, reações sorológicas da precipitina e de fixação do complemento (quantitativa) para diagnóstico da doença de Chagas, exame histológico de fragmento de músculo da panturrilha após coloração pela hematoxilina-eosina para procura de formas amastigotas do T. cruzi, eletrocardiograma e radiografia do tórax. Exames também realizados em 6 dos casos: inoculação de sangue no peritônio de 3 camundongos brancos, com aproximadamente $20 \mathrm{~g}$ de peso, para isolamento do T. cruzi, xenodiagnóstico nos camundongos com 5 ninfas de terceiro ou quarto estádios do T. infestans, hemograma, determinação da velocidade de hemossedimentação, dosagem da mucoproteína e das transaminases (glutâmico-oxalacética e glutâmico-pirúvica) no soro, prova da proteína reativa $\mathrm{C}$ com $\mathrm{O}$ soro e eletroforese das proteínas séricas. Cada camundongo recebeu $1 \mathrm{ml}$ de sangue com citrato de sódio a 3\%, suficiente para impedir coagulação. Sacrificamos os animais após 15 a 20 dias e, na ocasião, praticamos o xenodiagnóstico; histologicamente, por intermédio de coloração pela hematoxilinaeosina, houve procura do protozoário em músculos cardíaco e esquelético. Os exames laboratoriais inespecíficos foram realizados para ajuizar acerca de eventuais distúrbios colaterais e evidenciar, como marcadores indiretos, a atividade infecciosa-inflamatória.

Acompanhamento dos pacientes. Os exames foram realizados a cada 10 ou 15 dias e só ocasionalmente com intervalos de 20 a 30 , durante o tratamento, em número de 3 a 7 , mas, em geral, de 4 para cada caso. Cumpre assinalar que a biópsia de músculo da panturrilha foi realizada apenas uma vez $\left(30^{\circ}\right.$ dia de tratamento). Para cálculo estatístico foram considerados, como dias de tratamento, 0 (antes), 15, 30, 45 e 60 dias.

A quantidade total de xenodiagnósticos durante o tratamento correspondeu a 44 , englobando 857 triatomíneos vivos, e os referentes a camundongos somaram 14, abrangendo 64 insetos vivos.

\section{Grupo II}

Casuística. Composta de 10 pacientes (50\% do sexo masculino), nenhum deles apresentando afecção associada. As idades variaram de 18 a $44 \operatorname{anos}(29,4 \pm 9,2)$.

Exames auxiliares. Xenodiagnóstico com 20 ninfas de terceiro ou quarto estádios do $T$. infestans, reações sorológicas da precipitina e de fixação do complemento (quantitativa) para diagnóstico da doença de Chagas, inoculação de sangue no peritônio de 3 camundongos brancos para isolamento do T. cruzi, seguindo a mesma metodologia usada no Grupo I, xenodiagnóstico nos camundongos com 5 ninfas de terceiro ou quarto estádios do $T$. infestans, realizado por ocasião do sacrifício dos mesmos, eletrocardiograma e radiografia do tórax. 
Rassi A, Amato Neto V, Siqueira AF, Doles J, Leite MSB, Silva OQ, Cardoso VM. Influência de corticóide, na doença de Chagas crônica, administrado em virtude de afecções associadas. Revista da Sociedade Brasileira de Medicina Tropical 30:93-99, mar-abr, 1997.

Acompanhamento dos pacientes. Avaliações periódicas foram efetuadas a cada 15 dias e só ocasionalmente com intervalo de 30 , em número de 4 para cada paciente. A quantidade total de xenodiagnósticos correspondeu a 40 , englobando 780 triatomíneos vivos; os referentes a camundongos somaram 34, abrangendo 157 insetos vivos.

\section{Grupo III}

Casuística. Composta de 24 pacientes, 13 do sexo masculino e 11 do feminino. As idades variaram de 15 a 62 anos $(31,2 \pm 14,5)$. Diagnósticos das afecções associadas, que justificaram o uso de corticóide: pênfigo foliáceo (20), doença reumática (1), neurolues (1), polineuropatia iatrogênica devida ao benzonidazol (1) e púrpura trombocitopênica secundária em paciente com hiperesplenismo da leishmaniose visceral (1).

Uso de corticóide. As doses dos diferentes corticóides, computadas como de prednisona e calculadas como médias, variaram de 90 a $9,7 \mathrm{mg}$, com administração progressivamente decrescente, de 12 em 12 horas, e em etapas que duraram de 45 a 50 dias, com alguns extremos de 44 e 63.

Exames auxiliares. Reações sorológicas de imunofluorescência indireta e de fixação do complemento quantitativas para diagnóstico da doença de Chagas, xenodiagnóstico com 20 ninfas de terceiro ou quarto estádios do $T$. infestans ( 46 vezes) e com 30 (80 vezes) ou 40 (10 vezes), eletrocardiograma e radiografia do tórax.

Acompanhamento dos pacientes. Avaliações periódicas foram efetuadas a cada 7 ou 15 dias, em número de 3 a 8 , mas, em geral, de 3 para cada caso. A quantidade total de xenodiagnósticos correspondeu a 136, englobando 3.456 triatomíneos vivos.

Sem exceção, os insetos de todos os xenodiagnósticos, tanto os realizados nos pacientes como os aplicados nos camundongos, tiveram suas fezes individualmente examinadas cerca de 45 dias depois do repasto, com sacrifício dos mesmos. Os triatomíneos mortos foram excluídos da observação.

Durante a pesquisa, os enfermos dos Grupos I e III permaneceram internados e para os do II adotamos seguimento ambulatorial, em instituições sediadas em Goiânia, no Estado de Goiás (Hospital do Pênfigo - atual Hospital de Doenças Tropicais Dr. Anuar
Auad, Hospital das Clínicas da Faculdade de Medicina da Universidade Federal de Goiás, Hospital São Salvador e Hospital do Câncer atual Hospital Araújo Jorge).

Análise de dados. Foram calculadas as percentagens de triatomíneos positivos e respectivos intervalos de $95 \%$ de confiança para cada grupo de tratamento em cada seguimento (dias $0,15,30,45,60$ ). Para o cálculo das percentagens utilizou-se a fórmula:

$$
\frac{\mathrm{n}^{\mathrm{o}} \text { triatomíneos positivos }}{\mathrm{n}^{\mathrm{o}} \text { triatomíneos vivos }} \times 100
$$

O teste "t" e a análise de variância foram utilizados para avaliar as diferenças entre duas ou mais médias. O teste de qui-quadrado foi utilizado para avaliar diferenças observadas entre a distribuição de freqüência.

\section{RESULTADOS}

A Tabela 1 mostra que não houve diferença significativa entre as médias de idade e sexo entre os grupos. Por outro lado, as doses médias de corticóide utilizadas entre o $31^{\circ}$ e 45 dias nos Grupos I e III, foram estatisticamente diferentes $(p=0,02)$.

Tabela 1 - Características básicas dos pacientes.

\begin{tabular}{|c|c|c|c|}
\hline \multirow[b]{2}{*}{ Característica } & \multicolumn{2}{|c|}{ Grupo corticóide } & \multirow{2}{*}{$\begin{array}{c}\text { Grupo controle } \\
\text { II } \\
\mathrm{n}=10\end{array}$} \\
\hline & $\begin{array}{c}\mathrm{I} \\
\mathrm{n}=10\end{array}$ & $\begin{array}{c}\text { III } \\
\mathrm{n}=24\end{array}$ & \\
\hline \multicolumn{4}{|l|}{ Idade (anos) } \\
\hline média* & 37,6 & 31,2 & 29,4 \\
\hline desvio padrão & 16,1 & 14,5 & 9,2 \\
\hline Masculino (\%) & 60,0 & 54,0 & 50,0 \\
\hline Triatomíneos + dia $0(\%)^{* * *}$ & 29,8 & 8,1 & 2,1 \\
\hline \multicolumn{4}{|l|}{$\begin{array}{l}\text { Dose média/dia de corticóide } \\
\text { expressa em prednisona (mg) }\end{array}$} \\
\hline dia $1-15$ & 68 & 61 & - \\
\hline dia $16-30$ & 47 & 37 & - \\
\hline dia $31-45$ & 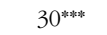 & 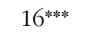 & - \\
\hline dia $46-60$ & 18 & - & - \\
\hline
\end{tabular}

Os resultados dos xenodiagnósticos realizados nos pacientes estão representados nas Figuras 1 e 2, correlacionados com a média das doses diárias de corticóide. Os Grupos II e III não diferiram quanto à \% de triatomíneos positivos no dia 0 , conforme se observa pela justaposição dos respectivos intervalos de confiança; por este motivo, puderam ser apresentados em um mesmo gráfico, para 
Rassi A, Amato Neto V, Siqueira AF, Doles J, Leite MSB, Silva OQ, Cardoso VM. Influência de corticóide, na doença de Chagas crônica, administrado em virtude de afecções associadas. Revista da Sociedade Brasileira de Medicina Tropical 30:93-99, mar-abr, 1997.

efeito de comparação. O mesmo não aconteceu com o Grupo I.

Histologicamente, nunca encontramos o parasita, nem no músculo da panturrilha dos pacientes e nem na musculatura estriada e cardíaca dos camundongos.
O xenodiagnóstico nos camundongos inoculados resultou positivo apenas em 2 de 6 casos do Grupo I.

A prova da precipitina teve comportamento extremamente variável e a desordenada verificação de positividades e negatividades no

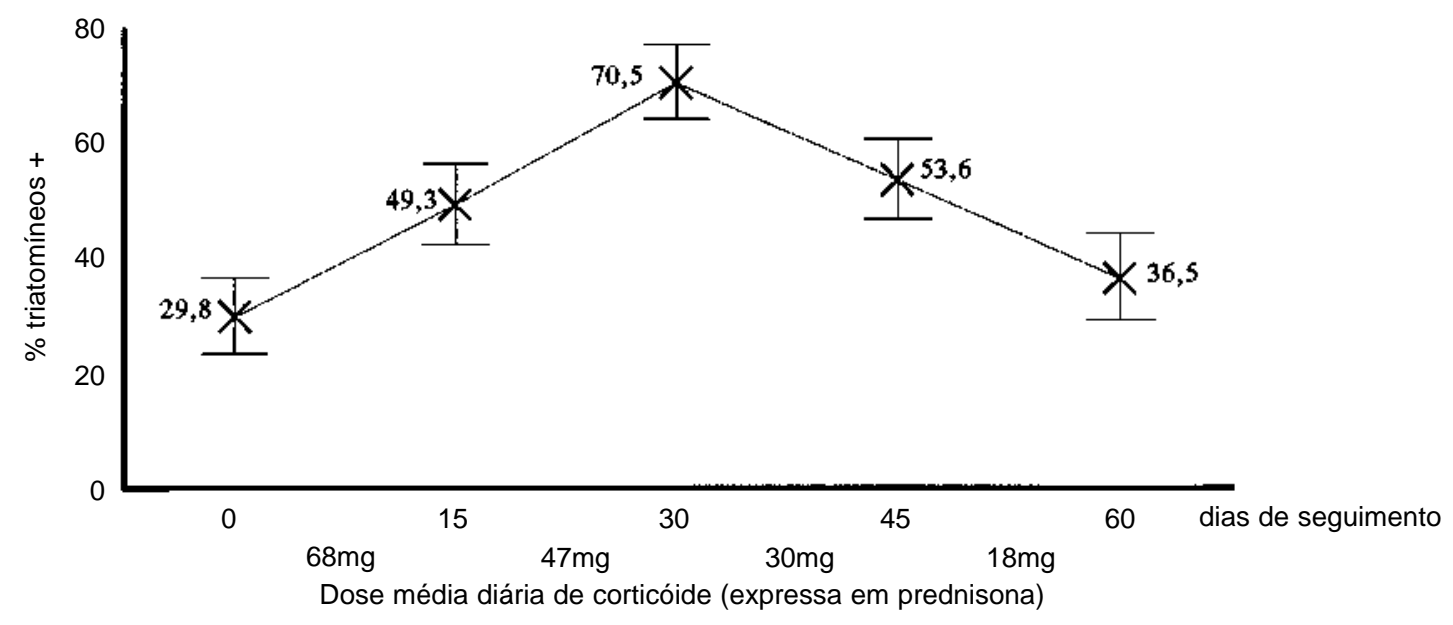

Figura 1 - Positividade de triatomíneos ao xenodiagnóstico em pacientes do Grupo I.

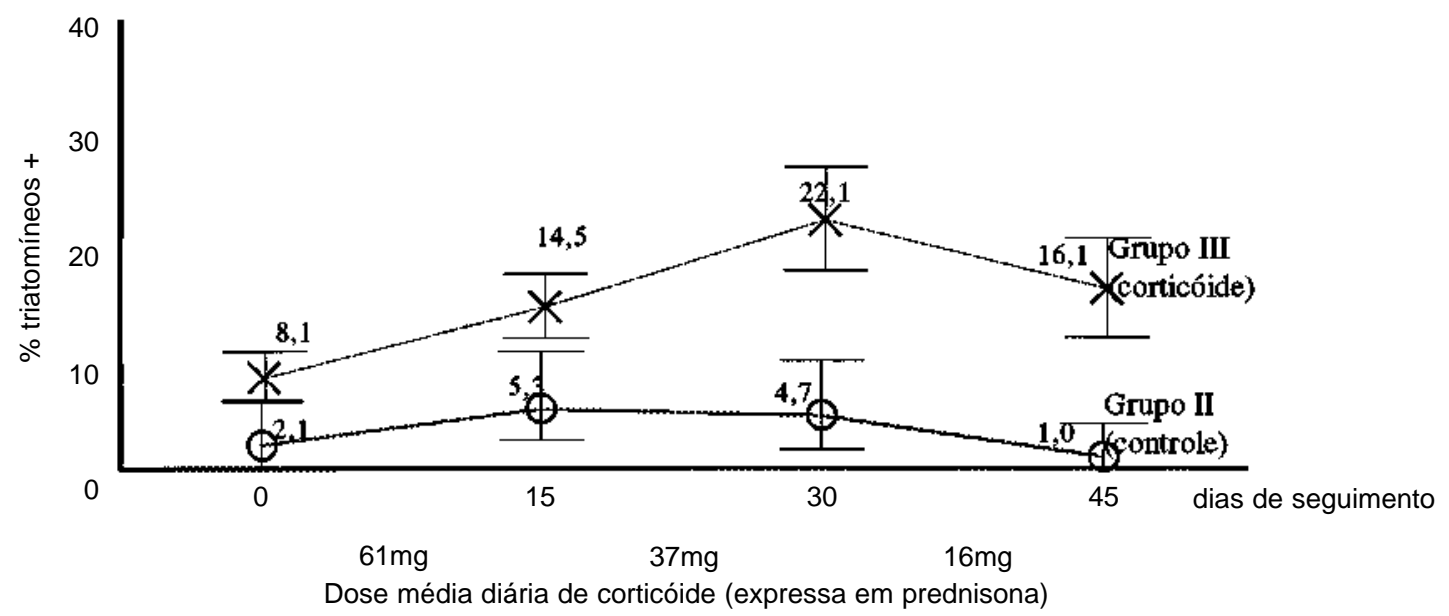

Figura 2 - Positividade de triatomíneos ao xenodiagnóstico em pacientes dos Grupos II e III. 
Rassi A, Amato Neto V, Siqueira AF, Doles J, Leite MSB, Silva OQ, Cardoso VM. Influência de corticóide, na doença de Chagas crônica, administrado em virtude de afecções associadas. Revista da Sociedade Brasileira de Medicina Tropical 30:93-99, mar-abr, 1997.

mesmo paciente impediu aproveitamento capaz de fornecer deduções aceitáveis.

O hemograma, as determinações da velocidade de hemossedimentação, das transaminases e mucoproteína séricas, juntamente com a prova da proteína reativa $\mathrm{C}$ e da eletroforese das proteínas do soro, praticados só no Grupo I, não identificaram claros danos tóxicos e apontaram resultados muito díspares e nada uniformes, deixando de fornecer subsídios efetivos.

O exame radiológico revelou discreto aumento da área cardíaca em apenas 1 caso (Grupo I), no $105^{\circ}$ dia de tratamento, no qual o eletrocardiograma, que mostrava extra-sistolia ventricular, permaneceu inalterado. Neste paciente, o xenodiagnóstico resultou negativo antes do tratamento e sempre positivo na vigência do mesmo, um deles, no $43^{\circ}$ dia de tratamento, com 19 dentre 20 (95\%) dos triatomíneos positivos; também resultou positivo 1 xenodiagnóstico realizado nos camundongos inoculados, com 4 dentre 5 (80\%) dos triatomíneos positivos.

O eletrocardiograma demonstrou acentuação de alteração da repolarização ventricular (ARV) pré-existente em 2 casos do Grupo I e em 1 do Grupo III, aparecimento de ARV em 1 caso do Grupo II e diminuição de ARV pré-existente em 1 do Grupo III. Em todos esses casos o resultado do xenodiagnóstico não foi diferente do observado nos pacientes em que o traçado permaneceu inalterado.

Sob o ponto de vista clínico, nenhuma modificação relativa à doença de Chagas pôde ser percebida no período em que se deu o estudo.

\section{DISCUSSÃO}

Para atingir o objetivo estipulado, ou seja, procurar ajudar a esclarecer como um medicamento imunodepressor, especificamente representado por corticóide, influi na infecção crônica pelo $T$. cruzi, marcadores de várias ordens compuseram o esquema avaliador, tendo ficado possível perceber que ele inclui amplo e trabalhoso conjunto de procedimentos, tidos como adequados.

Sem dúvida, foi decepcionante o comportamento da maioria dos processos utilizados, a despeito do vínculo que eles mantêm com a parasitose. Prova da precipitina, prestimosa no diagnóstico da fase aguda, aqui se mostrou de maneira muito heterogênea. As reações de imunofluorescência indireta e de fixação do complemento não trouxeram subsídios, igualmente porque seus resultados só revelaram oscilações temporais nunca consistentes, à semelhança do que ocorre espontaneamente. Inoculação e xenodiagnóstico nos camundongos inoculados, pelas constantes negatividades, outrossim, pouco ajudaram, apenas valendo lembrar as duas positividades anotadas no Grupo I. Ao contrário, o xenodiagnóstico efetuado nos pacientes de fato ajudou, mostrando maiores parasitemias nos Grupos I e III, ao ser levado em conta o observado no II. Constatamos diferenças estatisticamente significativas, que apóiam esta conclusão.

As Figuras 1 e 2 mostram aumento significativo da positividade de triatomíneos apenas nos pacientes que receberam corticóide, configurando uma curva ascendente-descendente com pico no $30^{\circ}$ dia de tratamento, diretamente relacionada com a dose do medicamento. Apesar da Tabela 1 mostrar diferença significante entre as doses médias de corticóide administradas do $31^{\circ}$ ao $45^{\circ}$ dia entre os Grupos I e III, esta diferença parece não ter exercido efeito diferencial nos resultados, uma vez que de acordo com a Figura 2, a \% de triatomíneos positivos no Grupo I já vinha apresentando tendência de queda a partir do $30^{\circ}$ dia, independente da dose administrada do $31^{\circ}$ ao $45^{\circ}$ dias.

Para formar juízo sobre a parasitemia na fase crônica da doença de Chagas, o xenodiagnóstico, inegavelmente, apesar de imperfeito e não possuidor da sensibilidade almejada, é valioso, enquanto não dispusermos de método melhor. Compete com a hemocultura que, contudo, esbarra em falta de desejável reprodutibilidade conforme as técnicas escolhidas e os laboratórios executores. Nesta investigação, o xenodiagnóstico possibilitou a percepção de que o corticóide conduziu a aumento do número de tripanossomos no sangue, circunstância obrigatoriamente digna de consideração, por exemplo, em ação terapêutica, transplante de órgão, administração de fármaco imunodepressor e presença de afecção no âmbito da qual déficit imunitário pode comparecer ${ }^{14} 15$.

Indiscutivelmente, o corticóide exacerbou a parasitemia, demarcada pelo xenodiagnóstico. Programamos e levamos a cabo plano avaliador 
Rassi A, Amato Neto V, Siqueira AF, Doles J, Leite MSB, Silva OQ, Cardoso VM. Influência de corticóide, na doença de Chagas crônica, administrado em virtude de afecções associadas. Revista da Sociedade Brasileira de Medicina Tropical 30:93-99, mar-abr, 1997.

amplo, para então notar a precária cooperação de diferentes recursos adotados. Relatamos tudo o que verificamos para ajudar os eventualmente interessados em empreender investigações semelhantes. Não obstante, o xenodiagnóstico contribuiu para demonstrar fato importante, válido para boa interpretação de ocorrências assistenciais, diagnósticas, patogenéticas e terapêuticas, entre outras. Períodos maiores de uso e doses mais elevadas quiçá desencadeiem repercussões de portes diversos, mas só novas especulações poderão esclarecer isso.

\section{SUMMARY}

Patients with chronic Chagas'disease and simultaneous medical problems treated with corticosteroid were studied in order to evaluate steroid influence on chronic Trypanosoma cruzi infection. Parasitological assessment, radiological and electrocardiographic studies as well as non specific tests were performed in patients and in a control group that included chronic infected patients not treated with steroid. Xenodiagnosis showed a clear increase in T. cruzi parasitemia, related to the corticosteroid dosage, without clinical manifestations during the study follow-up period.

Key-words: Chronic Chagas' disease. Corticosteroid therapy.

\section{AGRADECIMENTOS}

Os autores são gratos à Professora Ana Lúcia S.S. de Andrade pela análise estatística e à Professora Simonne A. Silva pela computação gráfica (Instituto de Patologia Tropical e Saúde Pública da Universidade Federal de Goiás).

\section{REFERÊNCIAS BIBLIOGRÁFICAS}

1. Abath FGC, Gomes YM, Coutinho EM, Montenegro SML, Melo MEB, Carvalho AB. Effects of betamethasone on the course of experimental infection with Trypanosoma cruzi. Revista da Sociedade Brasileira de Medicina Tropical 19:161$164,1986$.

2. Almeida HO, Tafuri WL, Bogliolo L, Cunha JC. Parasitismo incomum do miocárdio e do esôfago em chagásico crônico, portador de doença de Hodgkin e em uso de imunodepressores. Revista da Sociedade Brasileira de Medicina Tropical 8:117-121, 1974.

3. Andrade SG, Andrade ZA, Sadigursky M. Combined treatment with a nitrofuranic and a corticoid in experimental Chagas'disease in the dog. The American Journal of Tropical Medicine and Hygiene 29:766-773, 1980.

4. Bocchi EA. Transplante cardíaco em portadores da cardiopatia chagásica. Revista da Sociedade de Cardiologia do Estado de São Paulo 4:198-204, 1994.

5. Brener Z, Chiari E. The effects of some immunosuppressive agents in experimental chronic Chagas's disease. Transactions of the Royal Society of Tropical Medicine and Hygiene 65:629-636, 1971.

6. Chocair PR, Amato Neto V, Sabbaga E, Torrecillas $\mathrm{PH}$. Aspectos clínico-diagnósticos relativos à fase aguda da doença de Chagas, em pacientes submetidos à transplante de rim e imunodeprimidos. Revista da Sociedade Brasileira de Medicina Tropical 18:43-45, 1985.

7. Del Castillo M, Mendoza G, Oviedo J, Bianco RPP, Anselmo AE, Silva M. AIDS and Chagas' disease with central nervous system tumor-like lesion. The American Journal of Medicine 88:693-694, 1990.

8. Figueiredo JFC, Fiorillo AM, Ferraz AS. Longitudinal study of the indirect immunofluorescence and complement fixation tests for diagnosis of Chagas' disease in immunosuppressed patients submitted to renal transplantation. Revista da Sociedade Brasileira de Medicina Tropical 26:211-214, 1993.

9. França LCM, Fleury RN, Ramos Jr HA, Lemos S, Melaragno Filho R, Pasternak J. Moléstia de Chagas crônica associada a leucemia linfática: ocorrência de encefalite aguda como alteração do estado imunitário. Arquivos de NeuroPsiquiatria 27:59-66, 1969.

10. Kohl S, Pickering LK, Frankel LS, Yaeger RG. Reactivation of Chagas'disease during therapy of acute lymphocytic leukemia. Cancer 50:827-828, 1982.

11. Libow LF, Beltrani VP, Silvers DN, Grossman ME. Post-cardiac transplant reactivation of Chagas' disease diagnosed by skin biopsy. Cutis 48:37-40, 1991.

12. Okumura M, Amato Neto V, Kitagawa MM, Takamatsu NK, Ishikawa PS, Iizuza FH, Iriya K. Atividade terapêutica do benzonidazol em camundongos infectados pelo Trypanosoma cruzi e imunodeprimidos por associação de ciclosporina com prednisona. Revista do Hospital das Clínicas da Faculdade de Medicina da Universidade de São Paulo 45:260-262, 1990. 
Rassi A, Amato Neto V, Siqueira AF, Doles J, Leite MSB, Silva OQ, Cardoso VM. Influência de corticóide, na doença de Chagas crônica, administrado em virtude de afecções associadas. Revista da Sociedade Brasileira de Medicina Tropical 30:93-99, mar-abr, 1997.

13. Okumura M, Décourt LV. Estudo de efeitos da administração de drogas imunodepressoras sôbre a moléstia de Chagas experimental. Revista do Hospital das Clínicas da Faculdade de Medicina da Universidade de São Paulo 24:335-342, 1969.

14. Pizzi PT. Inmunologia de la enfermedad de Chagas: estado actual del problema. Boletin de la Oficina Sanitaria Panamericana 51:450-464, 1961.

15. Teixeira VPA, Reis MA, Araújo MBM, Silveira SA, Reis L, Almeida HO. Comparação do parasitismo da veia central da supra-renal com o de outros tecidos em chagásicos crônicos. Revista da Sociedade Brasileira de Medicina Tropical 24:7378, 1991.

16. Vichi FL, Albuquerque RD, Santos J, Soubihe NV, Moreira AC, Gil Netto J. The effect of oral treatment with prednisone on T.cruzi parasitemia of patients with chronic Chagas'heart disease. Revista do Instituto de Medicina Tropical de São Paulo 11:280-284, 1969. 